\title{
Nerve Tumors of the Upper Extremity
}

\author{
Sophia A. Strike, $M D^{a, *}$, Mark E. Puhaindran, MBBS, MMED (Surg) ${ }^{b}$
}

\section{KEYWORDS}

- Schwannoma $\bullet$ Neurilemmoma $\bullet$ Neurofibroma $\bullet$ Malignant peripheral nerve sheath tumor

- Neurofibromatosis

\section{KEY POINTS}

- Benign peripheral nerve sheath tumors are far more common than malignant peripheral nerve sheath tumors.

- Malignant transformation is more common when tumors are associated with neurofibromatosis.

- A rapid increase in size of a tumor or pain is suggestive of malignant transformation.

- Imaging characteristics suggestive of malignant nerve sheath tumors include larger size, perilesional edema, heterogeneous enhancement, and perilesional enhancement on MRI as well as a standardized uptake value greater than 4.0 on PET with fludeoxyglucose F 18.

- Surgical excision is the treatment of choice. Schwannomas are well encapsulated and typically can be separated from the underlying nerve with microscopic intrafascicular dissection whereas neurofibromas may require nerve excision due to their tendency to intertwine with nerve fascicles.

\section{INTRODUCTION}

Nerve tumors of the upper extremity include benign peripheral nerve sheath tumors (BPNSTs) and malignant peripheral nerve sheath tumors (MPNSTs). The most common peripheral nerve sheath tumors (PNSTs) of the hand and upper extremity are schwannomas, which are benign tumors of Schwann cell origin ${ }^{1-4}$ (Fig. 1). Multiple schwannomas may occur in schwannomatosis. Neurofibromas, also benign, are classically associated with neurofibromatosis (NF) but can occur as solitary lesions. These tumors also may be associated with gigantism of the underlying anatomy. ${ }^{1}$ BPNSTs can be observed or surgically excised with careful microscopic intrafascicular dissection. ${ }^{4}$ BPNSTs can transform to MPNSTs, which require more aggressive surgical treatment by a multidisciplinary team. Clinical and radiological factors for identifying malignant tumors and differentiating these from their benign counterparts are discussed. The most current recommended surgical techniques for tumor excision also are reviewed.

\section{CONTENT \\ Clinical Presentation}

Classic symptoms of malignant degeneration of an MPNST include rapid growth, pain at night, size greater than $5 \mathrm{~cm}$, previously soft consistency that becomes firm, and any associated constitutional symptoms. ${ }^{5}$ Malignant transformation is rare for schwannomas. ${ }^{1}$ Neurofibromas associated with NF are more likely to undergo malignant transformation than solitary lesions. ${ }^{1,2}$ MPNSTs may occur in $2 \%$ to $13 \%$ of patients with NF1 compared with $0.001 \%$ of the general population ${ }^{5,6} ; 50 \%$ of MPNSTs are in patients with NF1 but can occur postradiation or sporadically. ${ }^{7,8}$ Rapid growth of a plexiform neurofibroma in NF1 may not necessarily represent malignant transformation. This is a

Disclosure Statement: The authors have nothing to disclose.

a Department of Orthopaedic Surgery, Johns Hopkins University School of Medicine, Johns Hopkins Outpa-

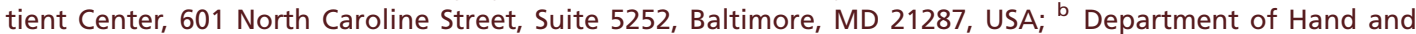
Reconstructive Microsurgery, National University Hospital, Level 11, NUHS Tower Block, 1E Kent Ridge Road, Singapore 11928, Singapore

* Corresponding author.

E-mail address: Sstrike1@jhmi.edu 


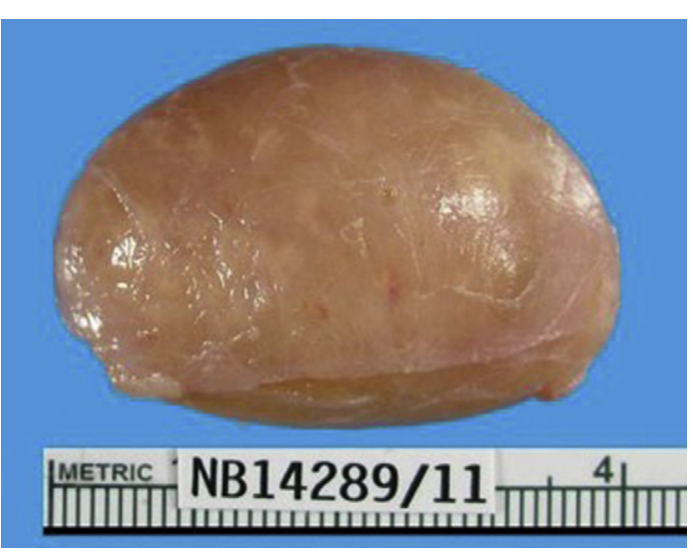

Fig. 1. Gross appearance of a schwannoma.

clinical finding that is still being actively studied. ${ }^{5} \mathrm{~A}$ recent study on the impact of family history suggests that a positive family history of MPNST is a risk factor for MPNST development at an earlier age in patients with $\mathrm{NF} 1{ }^{9}$

The typical clinical presentation of benign tumors is a firm, slow-growing mass that is either asymptomatic or causes radiating pain or paresthesia along the course of the nerve. ${ }^{1,2}$ On physical examination, a positive Tinel sign may be present over the mass. ${ }^{1}$

\section{Investigations}

Evaluation should begin with plain radiographs, which are likely to be normal in cases of most PNSTs. ${ }^{3}$ In general, a soft tissue mass of unknown character in the upper extremity that is concerning for a PNST requires further work-up with MRI because this is the most useful imaging modality for these tumors. ${ }^{3,10,11}$ Nerve sheath tumors classically are homogeneously hypointense centrally with a peripheral hyperintensity, known as the target sign, although this is not specific to nerve sheath tumors. ${ }^{11,12}$ Nilsson and colleagues ${ }^{10}$ reported MRI to be diagnostic in $75 \%$ of their series of nerve tumors of the upper extremity. In a study by Hung and colleagues, ${ }^{11}$ the diagnostic accuracy of ultrasound and of MRI in the evaluation of nerve tumors of the upper extremity was reported as $77 \%$ and $100 \%$, respectively. MPNSTs are more likely to be larger and have perilesional edema, heterogeneous enhancement, and perilesional enhancement. ${ }^{12}$ The target sign is more specifically associated with BPNSTs. ${ }^{12}$ Diffusionweighted imaging and apparent diffusion coefficient mapping can be used to help differentiate between benign and MPNSTs. ${ }^{12}$

The role of PET with fludeoxyglucose $F 18$ ( ${ }^{18} \mathrm{~F}$ FDG)-PET/CT in identifying PNSTs that have undergone malignant transformation is still being developed. ${ }^{5}$ A standardized uptake value (SUV) of 4.0 has been suggested as a cutoff to help distinguish between benign and MPNSTs. In a study of pediatric patients with NF1, sensitivity and specificity of 1.0 and 0.94 , respectively, were reported when using this SUV. ${ }^{13}$ PET-CT in conjunction with high-resolution MRI has been studied with potential objective features unique to MPNSTs. These technical imaging features may be more useful for radiologists in assisting surgeons with diagnosis. Serial ${ }^{18} \mathrm{~F}$ FDG-PET/CT scans have been used for monitoring lesions in patients with NF1 for malignant transformation. ${ }^{14}$

When clinical and radiological findings are not clearly consistent with a benign nerve sheath tumor, a core needle biopsy should be performed, although it can be painful. ${ }^{11}$ For a lesion without suspicious malignant characteristics and clinical and radiological findings of a benign nerve sheath tumor, a marginal excision can be performed.

\section{Management}

BPNSTs can be observed if they remain stable in size and asymptomatic. Surgical management of BPNSTs is with marginal excision. Due to their encapsulated nature, schwannomas are more amenable to intraneural dissection than neurofibromas, allowing preservation of the associated nerve. ${ }^{1-4}$ Intracapsular dissection and meticulous tumor removal under magnification preserve nerve structures and are recommended for schwannomas of the hand and wrist ${ }^{4,15}$ (Fig. 2). In a series of 14 patients treated with intracapsular dissection, no recurrences were reported at a mean follow-up of 12.6 years. ${ }^{15}$ Neurofibromas classically intertwine with the underlying nerve, and thus intraneural dissection and fascicular preservation may not be possible. ${ }^{1,2}$ If there is significant morbidity with resection of the associated nerve, it is reasonable to perform a biopsy to ensure the tumor is not malignant and then observe. If the affected nerve is not of functional importance, then tumor excision with primary nerve repair versus nerve grafting is the recommended treatment. ${ }^{1-3}$ Cutaneous nerves can be easily excised with sacrifice of the nerve. ${ }^{3}$ Intraneural dissection with microscopic magnification may be possible for neurofibromas associated with large nerves, which allows preservation of function. ${ }^{1,3}$ We prospectively studied patients with schwanommas and neurofibromas who underwent marginal excision using microscopic intrafascicular dissection and found, on histological analysis, that nerve fascicles may even run through the main tumor and may require sacrifice if a marginal excision is performed (Fig. 3). Plexiform neurofibromas, which 

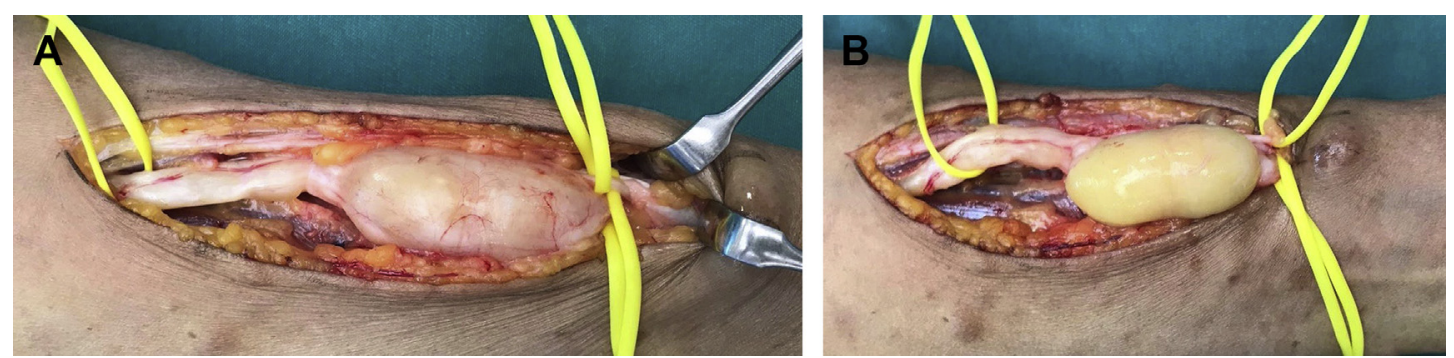

Fig. 2. Neurofibroma in a patient with NF1, before $(A)$ and after $(B)$ microscopic intraneutral intrafascicular dissection.

are almost exclusively associated with NF1, typically are difficult to separate from the surrounding nerve. ${ }^{16}$ In a recent review of a large series of 62 patients with NF1 and plexiform neurofibromas of the upper extremity and hand, a majority of patients underwent 3 or fewer surgical procedures for these tumors and $74 \%$ of the procedures had no complications, with only $0.6 \%$ of procedures resulting in a temporary sensibility disorder. ${ }^{16}$ Postoperative neurologic disorder usually is temporary but has been reported to occur in up to $50 \%$ of patients and patients should be counseled accordingly. ${ }^{11}$

MPNSTs require wide excision. Nerve reconstruction may not be helpful, and amputation should be considered for large or recurrent tumors. ${ }^{5,7}$ Radiotherapy for MPNSTs may provide local control but has not been shown to prolong survival. ${ }^{5,7}$ Chemotherapy generally is reserved for metastatic disease, although its role in MPNST management has not been fully elucidated. ${ }^{5,7}$ Tyrosine kinase inhibitors and anti-RAS pathway drugs may show some promise in management of MPSNTs. ${ }^{5}$

Percutaneous radiofrequency ablation has been described with reasonable success in the treatment of benign and MPNSTs in patients who are

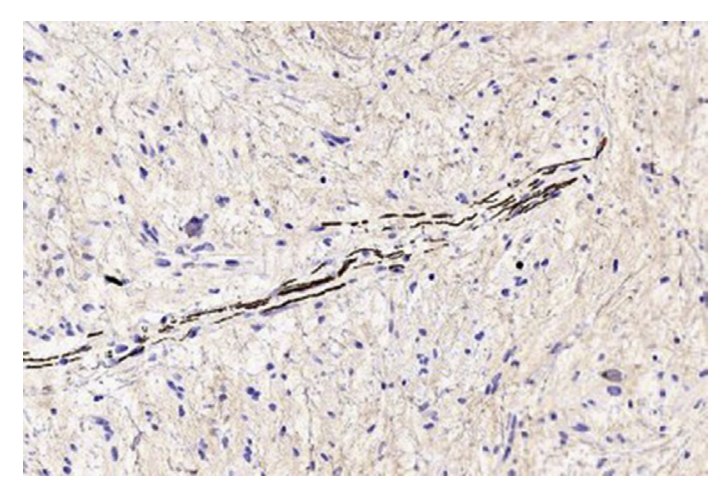

Fig. 3. Neurofilament stain demonstrating nerve fiber running through lesion near periphery (immunohistochemistry, $\times 250$ ). poor surgical candidates. ${ }^{17}$ This treatment modality has not been studied in the upper extremity. The anatomy of the upper extremity is more amenable to local surgical excision than the retroperitoneal PNSTs for which this technique has primarily been used. ${ }^{17}$

Recurrence after excision of a schwannoma is relatively rare. ${ }^{1,12}$ MPNSTs have a poorer prognosis, with $50 \%$ of patients presenting with metastases and 5 -year survival rates ranging from $15 \%$ to $50 \%{ }^{7}$

\section{SUMMARY}

BPNSTs and MPNSTs in the upper extremity can occur as solitary or as syndrome-related lesions. Classically, Tinel sign is positive over the tumor. Clinical features of rapid growth, new neuro- logic symptoms, and increased pain are suggestive of but not specific for malignant transformation. Radiological evaluation with MRI can provide diagnostic information in $75 \%$ of cases and ${ }^{18} \mathrm{~F} \mathrm{FDG-}$ PET/CT may be used to help differentiate benign from malignant lesions, with an SUV greater than 4.0 suggestive of malignant lesions. Treatment in the upper extremity is with surgical excision. Intraneural dissection is recommended for all schwannomas and many neurofibromas, although some degree of fascicular sacrifice may be necessary and the degree of excision should be tailored to the nerve involved. MPNSTs have a poor prognosis and require multidisciplinary care and a wide surgical excision, which may lead to amputation.

\section{REFERENCES}

1. Hsu CS, Hentz VR, Yao J. Tumours of the hand. Lancet Oncol 2007;8:157-66.

2. Longhurst W, Khachemoune A. An unknown mass: the differential diagnosis of digit tumors. Int J Dermatol 2015;54:1214-25.

3. Payne WT, Merrell G. Benign bony and soft tissue tumors of the hand. J Hand Surg 2010;35A:1901-10. 
4. Lai CS, Chen IC, Lan HC, et al. Management of extremity neurilemmomas: clinical series and literature review. Ann Plast Surg 2013;71(Suppl 1):S37-42.

5. Ferner RE1, Gutmann DH. International consensus statement on malignant peripheral nerve sheath tumors in neurofibromatosis. Cancer Res 2002;62(5): 1573-7.

6. Ducatman BS, Scheithauer BW, Piepgras DG, et al. Malignant peripheral nerve sheath tumors. A clinicopathologic study of 120 cases. Cancer 1986;57(10): 2006-21.

7. Farid M, Demicco EG, Garcia R, et al. Malignant peripheral nerve sheath tumors. Oncologist 2014; 19(2):193-201.

8. Longo JF, Weber SM, Turner-Ivey BP, et al. Recent advances in the diagnosis and pathogenesis of neurofibromatosis type 1 (NF1)-associated peripheral nervous system neoplasms. Adv Anat Pathol 2018;25(5):353-68.

9. Malbari F, Spira M, Knight PB, et al. Malignant peripheral nerve sheath tumors in neurofibromatosis: impact of family history. J Pediatr Hematol Oncol 2018;40(6):e359-63.

10. Nilsson J, Sandberg K, Søe Nielsen N, et al. Magnetic resonance imaging of peripheral nerve tumours in the upper extremity. Scand J Plast Reconstr Surg Hand Surg 2009;43(3):153-9.

11. Hung YW, Tse WL, Cheng HS, et al. Surgical excision for challenging upper limb nerve sheath tumours: a single centre retrospective review of treatment results. Hong Kong Med J 2010;16: 287 Y291.

12. Ahlawat S, Fayad LM. Imaging cellularity in benign and malignant peripheral nerve sheath tumors: utility of the "target sign" by diffusion weighted imaging. Eur J Radiol 2018;102:195-201.

13. Tsai LL, Drubach L, Fahey F, et al. [18F]-Fluorodeoxyglucose positron emission tomography in children with neurofibromatosis type 1 and plexiform neurofibromas: correlation with malignant transformation. J Neurooncol 2012;108(3):469-75.

14. Ren J, Yang G, Zhou J, et al. The value of 18F-FDG PET/CT in patient with neurofibromatosis type 1: a case report and literature review. Medicine 2018; 97:20.

15. Ozdemir O, Ozsoy MH, Kurt C, et al. Schwannomas of the hand and wrist: long-term results and review of the literature. J Orthop Surg (Hong Kong) 2005; 13(3):267-72.

16. Friedrich RE, Diekmeier C. Peripheral nerve sheath tumors of the upper extremity and hand in patients with neurofibromatosis type 1: topography of tumors and evaluation of surgical treatment in 62 patients. GMS Interdiscip Plast Reconstr Surg DGPW 2017; 6:Doc15.

17. Mrowczynski O, Mau C, Nguyen DT, et al. Percutaneous radiofrequency ablation for the treatment of peripheral nerve sheath tumors: a case report and review of the literature. Cureus 2018;10(4): e2534. 